\title{
The Use Of Miller Broach During Microsonic Removal Of Separated Endodontic File: Case Report
}

\author{
Karoly Schreindorfer ${ }^{*}$, Balint Tordai, Karoly Krajczar
}

DMD Dental School, Faculty of Medicine, University of Pecs, Hungary

*Corresponding author: Karoly Schreindorfer, Dental School, Faculty of Medicine, University of Pécs, H-7621 Pécs, DischkaGy. u. 5, Hungary, Tel: +003672535920; Fax: 003672535905; E-mail: sch.karesz@gmail.com

Received date: January 10, 2017; Accepted date: January 17, 2017; Published date: January 23, 2017

Copyright: ( 2017 Schreindorfer K. This is an open-access article distributed under the terms of the Creative Commons Attribution License, which permits unrestricted use, distribution, and reproduction in any medium, provided the original author and source are credited.

\begin{abstract}
Introduction: The microsonic technique is widely used for separated endodontic instrument removal.
\end{abstract}

Aim: We evaluated the usability of the Miller broach with the microsonic technique during separated instrument retrieval.

Case report: An attempt was made to remove a fractured instrument using a modified Miller needle mounted in a piezoelectric scaler under the visualization of an operating microscope. The conditions of the procedure were noted. The removal was successful. Severe preparation error did not revealed.

Discussion: The Miller needle have the properties for use in a modified microsonic technique. It can be bent to the individual situation and the preparation is considerable on the tip of the instrument. The procedure can be wellcontrolled and the operator may avoid excessive dentin removal.

Conclusion and clinical relevance: The Miller broach fulfills the requirements of the microsonic technique and have financial advantages.

Keywords: Case report; Broken endodontic instrument; Microsonic technique; Miller needle

\section{Introduction}

The fracture of endodontic instruments is a frequent complication during root canal treatment [1]. The fragment might inhibit proper root canal treatment beyond the fracture site thus possibly leading to failure [2]. Numerous instrument removal techniques have been discussed in the literature [3]. Certainly the most widely used and safest method is preparation with ultrasonic endodontic tips under direct visual control using a dental microscope (microsonic technique) [4].

Different types of ultrasonic instruments are available for removing separated endodontic files, thus the operator's preference can be an important factor in the selection. Materials of the ultrasonic instrument may vary among stainless steel, titanium, zirconium nitride coated stainless steel, and diamond-coated stainless steel (Figure 1). Either stainless steel or nickel-titanium ultrasonic endodontic files can be used with the help of a 95 or 120 degree contra-angled file holder. With the pre-bending of stainless steel files it is possible to work in curved canals, and with a slight bending of the file tip the direction of the preparation can be well controlled [5]. If ultrasonic files are mounted in the file holders, the remaining part of the files are around $25 \mathrm{~mm}$, as in the case of $25 \mathrm{~mm}$-long hand files. The use of longer instruments and the proper bending of the files may enhance visual control in root canals during the ultrasonic preparation.
We were looking for an alternative instrument that is longer than the conventional ultrasonic files, has no cutting edges and can be mounted in the ultrasonic file holders. We found that the Miller needle provides an alternative choice.

The Miller needle is an endodontic instrument used primarily to detect canals and hold cotton pellets during retrograde drying. It is manufactured with circular, triangular or pentagonal cross-sections.

\section{Aim}

Our purpose was to evaluate the usability of the Miller needle during a microsonic file removal procedure.

\section{Case Report}

A 28-year-old male patient reported to our public clinic. The patient was in good general health with no significant illness. From the referral it was revealed that his general dental practitioner (GDP) began endodontic treatment of 1.6 but a hand file separated during the procedure. On his visit to our clinic the patient's chief complaint was intermittent pain when chewing.

Clinical examination was performed: Tooth 1.6 had a mesioocclusal temporary filling, showed tenderness to vertical percussion and proved to be non-vital; periodontal probing depth was in normal range $(<3 \mathrm{~mm})$, and mobility was physiological. 


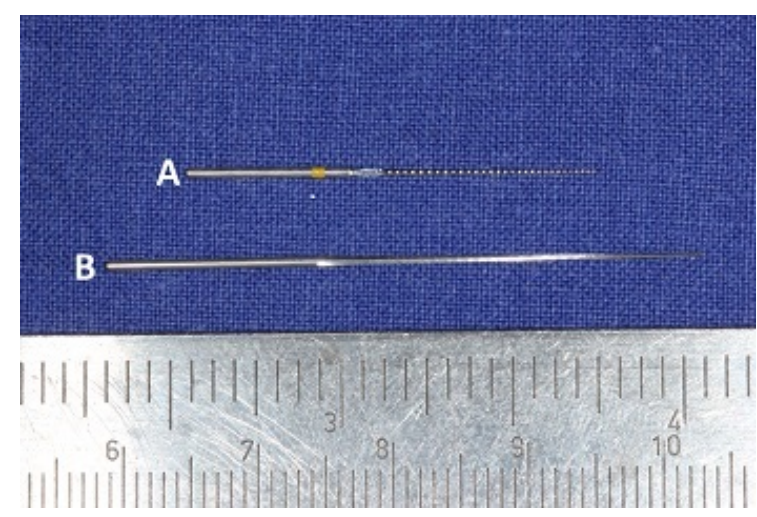

Figure 1: (A) Ultrasonic \#20 K-file and (B) Miller needle which was used during the removal.

The periapical radiograph presented a $4 \mathrm{~mm}$-long instrument fragment in the mesiobuccal root (Figure 2). The instrument was separated in the curvature of the root canal, which equaled the middle canal section. Around the apex of the mesiobuccal root, apical widening of the periodontal ligament was noted. Based on the clinical and radiological signs and symptoms, symptomatic periapical periodontitis was diagnosed.

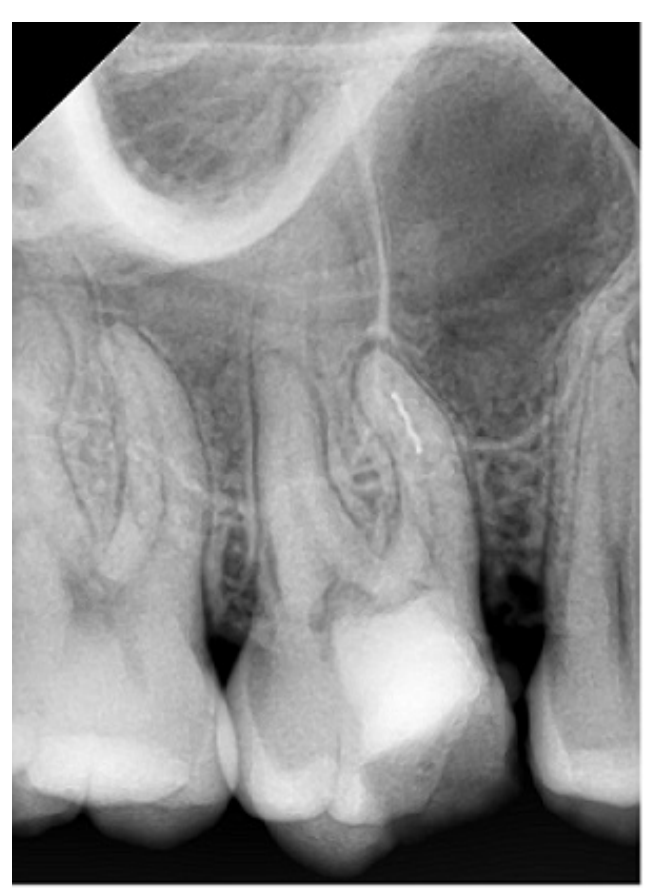

Figure 2: Preoperative periapical radiograph: separated instrument in the mesiobuccal root canal.

According to the literature, the removal or the bypassing of the separated file was considered as an ideal treatment option to achieve adequate chemomechanical disinfection and an attempt was taken to remove the fragment [6]. The operator decided to use the microsonic technique with some modifications for the removal of the fragment
[7]. Treatment planning, revision of the trepanation cavity, instrument removal, chemomechanical preparation, definitive root canal filling and the coronal filling were carried out in one single visit. The treatment was done in absolute isolation using a rubber dam.

The access cavity was modified and revised under dental microscope. Besides the previously treated mesiobuccal first (MB1), distobuccal (DB) and palatal (P) canals, the untreated second mesiobuccal root canal (MB2) was found. By the MB2 canal \#8, \#10 and \#15 stainless steel K-files were used to negotiate the root canal. Working length was measured with \#15 stainless steel K-file in the $\mathrm{MB} 2, \mathrm{DB}$ and $\mathrm{P}$ canals using an electronic apex locator (Raypex 6, VDW, Munich, Germany).

The preparation of the MB2, DB, and P root canals were carried out with R25 Reciproc files (VDW, Munich, Germany) using a Reciproc Silver endodontic engine (VDW, Munich, Germany). Each canal was copiously irrigated with a total amount of at least $5 \mathrm{ml} 2.5 \% \mathrm{NaOCl}$ solution during the mechanical preparation.

In the MB1 root canal the retrieval of the separated file was carried out using a modified microsonic technique. Straight-line coronal and radicular access was created to maintain appropriate visibility during further steps of removal. The MB1 canal was pre-enlarged (radicular access) coronally to the fragment using \#3 and \#2 Gates Glidden drills. Then a staging platform was created at the level of the separated file using a modified \#2 Gates Glidden drill (Figure 3) [5]. Thereafter the top of the separated file was direct visible with high magnifications (Figure 4).

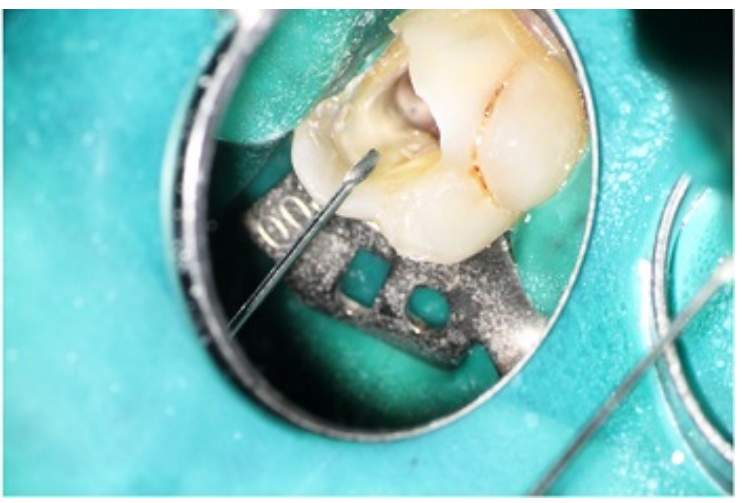

Figure 3. Modified Gates-Glidden bur used to create the staging platform.

The next step was the ultrasonic preparation with a piezoelectric scaler (UDS-E, Woodpecker, Guangxi, China). For the ultrasonic preparation we used a modified Miller needle (Dentsply Maillefer, Ballaigues, Switzerland) mounted in a 120 degree file holder (E1, Woodpecker, Guangxi, China) (Figure 5). Modification of the Miller broach consisted of two steps. First the instrument was bent according to the individual anatomic situation and for optimal access, and then the tip of the Miller needle was sharpened using a diamond bur. With these alterations the Miller needle was used to remove dentin around the separated fragment. During the procedure the debris was flushed out using $2.5 \% \mathrm{NaOCl}$ solution and the canal was dried with sterile paper points to improve visibility. The loosened fragment was agitated with the Miller needle until it was removed from the root canal. The removal of the instrument was successful (Figure 6). 


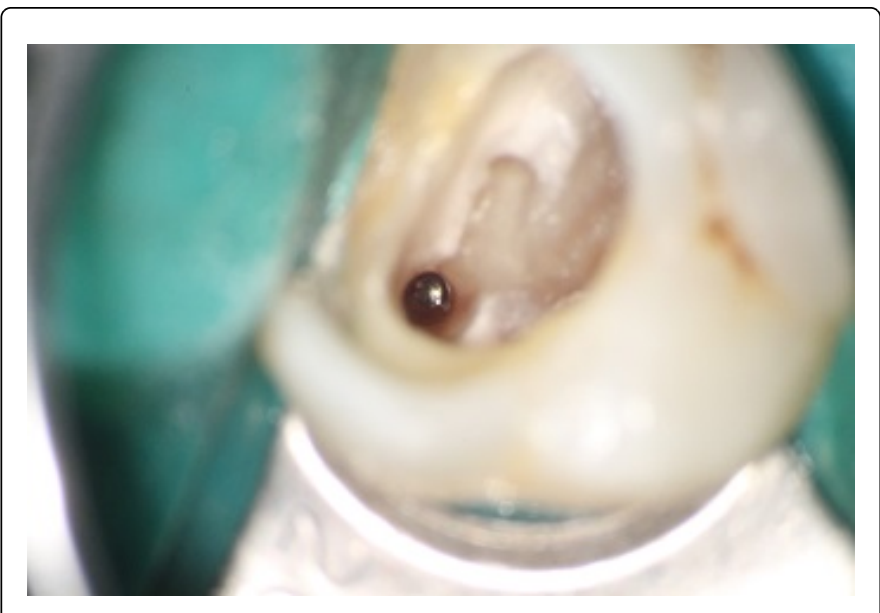

Figure 4: The top of the separated instrument.

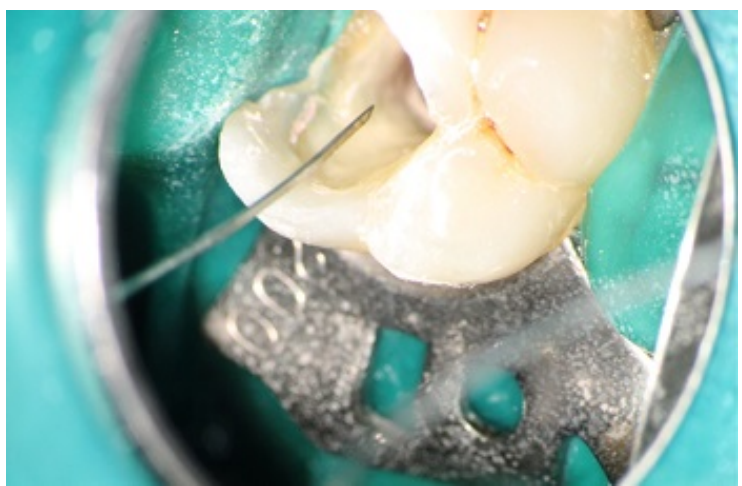

Figure 5: Bended and sharpened Miller needle before ultrasonic preparation.

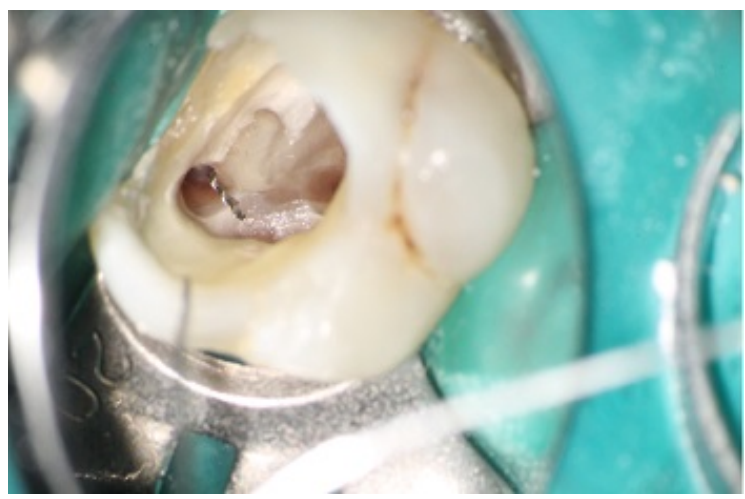

Figure 6: Unlocked instrument fragment in the pulp chamber.

Working length was determined by the MB1 canal with an electronic apex locator using a \#15 K-file. The final preparation of the root canal was made with an R25 Reciproc-file. Before the obturation the following irrigation protocol was used in all root canals:
First all of the four canals were copiously irrigated with $2.5 \% \mathrm{NaOCl}$ solution and the disinfectant was agitated with passive ultrasonic activation three times, each 20 seconds long [8]. Thereafter an intermediate washing was made with sterile distilled water. Then $18 \%$ EDTA (Ultradent Producs. Inc., South Jordan, UT, USA) solution was used for 1 minute. After the use of the EDTA solution intermediate washing took place with sterile distilled water. The final irrigation was made with the $2.5 \% \mathrm{NaOCl}$ solution.

All four canals were dried with paper points and obturated using 0.02 tapered gutta-percha cones and AH Plus sealer (Dentsply DeTrey, Konstanz, Germany) using the cold lateral condensation technique. A control periapical radiograph was taken to evaluate the root filling and the preparation form.

The access cavity was filled using light-curing composite material (Filtek Z250, 3M ESPE, St. Paul, MN, USA). The patient was recalled after 3, 6, 12 and 18 months.

The quality of root canal filling was rated ideal. There was no evidence of preparation errors, so often reported in such cases [9]. During and after the treatment the patient did not complain about discomfort or pain. During the recall period no clinical signs or symptoms were noticed. The success of the treatment was radiologically confirmed by an 18-month follow-up X-ray, which showed complete periapical healing (Figure 7).

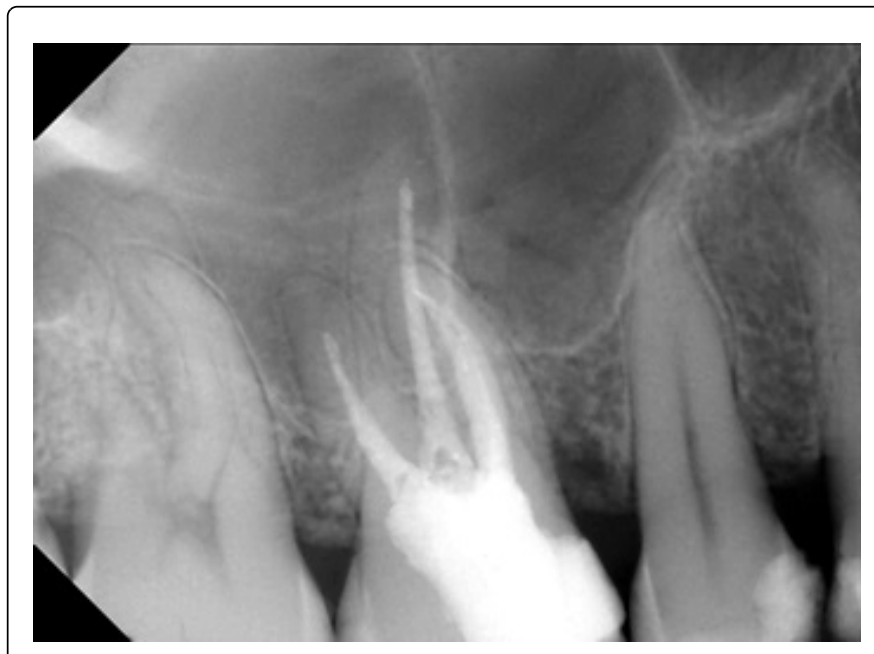

Figure 7: Control periapical radiograph: 18-month follow-up.

\section{Discussion}

This case report presented an entirely new application for the Miller needle. The Miller needle can be bent according to the individual anatomical situation, hence the visible operation field could be increased, and due to the better visual control, the preparation could be more accurate. Furthermore, the Miller broach has no sharp cutting surface, therefore dentin removal can be accomplished with the tip of the instrument. This enables the operator to avoid excessive dentin removal from the lateral walls.

Similarly to other ultrasonic tips, the Miller needle can fracture during the procedure. This additional accident can hinder the removal. Based on the operator's clinical experience, the likelihood of fracture seems to be correlated with the output power of the ultrasonic device. 
Citation: Schreindorfer K, Tordai B and Krajczar K (2017) The Use Of Miller Broach During Microsonic Removal Of Separated Endodontic File:

The temperature rise on the external root surface is also correlated to the output power [10]. To avoid these, we used our ultrasonic device only on endodontic (E) grade 1-5 (maximum power=10) for 1 minute continuously. The root canal was copiously irrigated with $2.5 \% \mathrm{NaOCl}$ solution to avoid heat stress. A fractured Miller broach does not usually wedge in the root canal. Nevertheless, if this happens it can be easily removed or flushed and vibrated out with irrigation material and an ultrasonic instrument.

\section{Conclusion and Clinical Relevance}

Based on the success of this particular case we have used this modified microsonic technique for broken instrument removal in more than 30 cases. Our overall success rate approximates the results reported by other studies. Compared to other ultrasonic instruments the Miller needle has a financial advantage and the removal procedure is safer for the patient and comfortable for the dentist owing to the increased visual control. With these benefits the microsonic technique combined with the Miller needle seems to be adequate to perform a minimally invasive instrument removal.

Our initial impressions are hopeful, but further investigations must be made to compare this with other techniques.

\section{Conflicts of Interest}

None to declare

\section{References}

1. Spili P, Parashos P, Messer HH (2005) The impact of instrument fracture on outcome of endodontic treatment. Journal of Endodontics 31: 845-850.
2. Madarati AA, Hunter MJ, Dummer PMH (2013) Management of intracanal separated instruments. Journal of Endodontics 39: 569-581.

3. Gencoglu N, Helvacioglu D (2009) Comprarison of the different techniques to remove fractured endodontic instruments from root canal systems. European Journal of Dentistry 3: 90-95.

4. Madarati AA, Watts DC, Qualtrough AJ (2008) Opinions and attitudes of endodontists and general dental practitioners in the UK towards the intracanal fracture of endodontic instruments: part 1. International Endodontic Journal 41: 693-701.

5. Plotino G, Pameijer CH, Grande NM (2007) Ultrasonics in endodontics: a review of the literature. Journal of Endodontics 33: 81-95.

6. Madarati AA (2016) Retrieval of multiple separated endodontic instruments using ultrasonic urlvibration: Case report. Journal of Taibah University Medical Sciences. Journal of Taibah University Medical Sciences 11: 268-273.

7. Ruddle CJ (1997) Micro endodontics: eliminating intracanal obstructions. Oral Health. 87: 19-24.

8. Cheung GS, Stock CJ (1993) In vitro cleaning ability of root canal irrigants with and without endosonics. International Endodontic Journal. 26: 334-343.

9. Santos SMC, Soares JA, Costa GM (2010) Radiographic parameters of quality of root canal fillings and periapical status: a retrospective cohort study. Journal of Endodontics. 36: 1932-1937.

10. Madarati AA, Qualtrough AJ, Watts DC (2008) Factors affecting temperature rise on the external root surface during ultrasonic retrieval of intracanal separated files. Journal of Endodontics 34: 1089-1092. 